\title{
Seleção de epífitas acumuladoras de elementos químicos na Mata Atlântica
}

\author{
Camila Elias, Elisabete A. De Nadai Fernandes, Elvis Joacir De França \& Márcio A. Bacchi
}

Biota Neotropica v6 (n1) - http://www.biotaneotropica.org.br/v6n1/pt/abstract?article+bn02106012006

Recebido em 11/08/05

Versão Modificada recebida 15/01/2006

Publicado em 17/02/06

Centro de Energia Nuclear na Agricultura, Universidade de São Paulo, Caixa Postal 96, 13400-970 Piracicaba, SP, Brasil.

\begin{abstract}
Elias, C.; De Nadai Fernandes, E.A.; França, E.J. and Bacchi, M.A. Selection of epiphytes as accumulators of chemical elements in the Atlantic Forest. Biota Neotrop. Jan/Abr 2006 vol. 6, no. 1, http://www.biotaneotropica.org.br/v6n1/pt/ abstract?article+bn02106012006. ISSN 1676-0603

Epiphytes are efficient indicators of atmospheric pollution because of their direct uptake of chemical elements from atmosphere. Leaves from eleven species of bromeliads and one species of orchid were collected in the Parque Estadual Carlos Botelho, SP, for determination of sixteen chemical elements. The selection of the best biomonitor species was based on the diversity and accumulation of chemical elements in the leaves. With an average accumulation index of 0.7 , Canistropsis billbergioides has potential to be used as a biomonitor of chemical elements in the Atlantic Forest.
\end{abstract}

Key words: INAA, Atlantic Forest, bromeliads, epiphytes, chemical elements

\section{Resumo}

Elias, C.; De Nadai Fernandes, E.A.; França, E.J. and Bacchi, M.A. Seleção de epífitas acumuladoras de elementos químicos na Mata Atlântica. Biota Neotrop. Jan/Abr 2006 vol. 6, no. 1, http://www.biotaneotropica.org.br/v6n1/pt/ abstract?article+bn02106012006. ISSN 1676-0603

Epífitas são eficientes indicadores de poluição atmosférica devido à absorção de elementos químicos diretamente da atmosfera. Folhas de onze espécies de bromélias e uma espécie de orquídea foram coletadas no Parque Estadual Carlos Botelho, SP, para a determinação de dezesseis elementos químicos. A seleção foi baseada na diversidade e acumulação de elementos químicos encontrados nas folhas. Com um índice de acumulação de 0,7, a espécie Canistropsis billbergioides tem potencial para ser empregada como biomonitora de elementos químicos na Mata Atlântica.

Palavras-chave: INAA, Mata Atlântica, bromélias, epífitas, elementos químicos

http://www.biotaneotropica.org.br 


\section{Introdução}

Organismos biomonitores podem ser utilizados para avaliar o impacto da poluição atmosférica em ecossistemas, permitindo estudar amplas áreas com a vantagem de esforço amostral reduzido (Wolterbeek 2002). Para tal avaliação, espécies podem ser selecionadas a partir da capacidade acumuladora de elementos químicos (Luoma \& Rainbow 2005). Abiomonitração pode ser passiva, quando o organismo empregado é nativo da área a ser avaliada, ou ativa, quando o biomonitor é transferido para o local (Markert 1991).

Organismos epifíticos não apresentam contato direto com o solo e formam um compartimento especial do ecossistema no contexto de estudos de ciclagem de elementos químicos. No caso de florestas tropicais, a aplicação desses organismos é recomendada dada a grande diversidade de espécies epifíticas, incluindo as famílias Bromeliaceae e Orchidaceae. Dentre as espécies biomonitoras de poluição, a bromeliácea Tillandsia usneoides já vem sendo utilizada, principalmente devido à sua adaptação morfológica para retirada de nutrientes da atmosfera por meio de escamas (Calasans et al. 1997, Pyat et al. 1999, Figueiredo et al. 2001). De acordo com os levantamentos fitossociológicos realizados nos principais tipos vegetacionais estudados no Projeto Temático BIOTA “Diversidade, Dinâmica e Conservação em Florestas do Estado de São Paulo: 40 ha de Parcelas Permanentes”, a família Bromeliaceae é uma das mais freqüentes, apresentando grande diversidade de espécies, principalmente na Parcela Permanente do Parque Estadual Carlos Botelho PECB (Breier et al. 2002).

O caráter conservacionista da pesquisa em parcelas permanentes não recomenda a introdução de uma espécie exótica como Tillandsia usneoides, já que essa espécie não ocorre naturalmente no interior da floresta (Breier et al. 2002). Aproveitando-se da biodiversidade existente para gerar conhecimentos a serem revertidos para a sua própria preservação, a seleção de epífitas nativas da região com capacidade de acumulação promove a sustentabilidade da pesquisa científica em unidades de conservação, possibilitando seu emprego em estudos de biomonitoração de elementos químicos em ecossistemas naturais. Baseandose na biomonitoração empregando folhas das espécies arbóreas mais relevantes, baixo nível de poluição foi encontrado na Parcela Permanente do PECB, embora algumas árvores tenham apresentado concentrações apreciáveis de elementos relacionados com a poluição atmosférica, como é o caso de bromo, cobalto e zinco (França et al. 2004). Desse modo, o presente trabalho teve como objetivo selecionar espécie epifítica nativa da Parcela Permanente do PECB com capacidade de acumulação de elementos químicos.

\section{Material e Métodos}

No Núcleo Sete Barras do PECB, uma parcela permanente de 10 ha foi instalada no contexto do projeto temático. Espécies epifíticas foram estudadas e identificadas no interior da Parcela Permanente (Breier et al. 2002) permitindo a utilização dos dados fitossociológicos para o desenvolvimento deste trabalho.

Foram amostrados 23 indivíduos completos de epífitas (Tabela 1) pertencentes à família Bromeliaceae (11 espécies) e à família Orchidaceae (1 espécie) no final do período fértil nas trilhas perimetrais da parcela permanente. Tipicamente, as bromélias estavam localizadas no subbosque, sendo também encontradas em clareiras naturais. A amostragem foi efetuada no entorno da área, de modo a não retirar indivíduos da parcela permanente. Paralelamente, pranchas com o material coletado foram preparadas para posterior identificação no herbário da Escola Superior de Agricultura Luiz de Queiroz (ESALQ/USP). Algumas espécies tiveram mais indivíduos coletados, como é o caso de Canistropsis billbergioides e Billbergia amoema, reflexo de suas abundâncias na área.

Todas as folhas foram lavadas com água de torneira, seguindo-se secagem em estufa à $60^{\circ} \mathrm{C}$ até peso constante e redução de granulometria (0,5 mm) em moinho de rotor de titânio. Porções das amostras com aproximadamente 200 mg foram transferidas para cápsulas de polietileno de elevada pureza, fabricadas pela Vrije Universiteit, Amsterdã. A técnica de análise por ativação neutrônica instrumental (INAA) foi empregada na avaliação da composição química. A INAA é baseada no bombardeamento da amostra com nêutrons para a produção de radionuclídeos, sendo que a medição da radioatividade induzida permite a quantificação dos elementos. Para avaliar a qualidade do procedimento analítico, adicionaram-se porções de $300 \mathrm{mg}$ dos materiais de referência certificados IAEA-336 Lichen e INCT-TL-1 Tea Leaves. Como monitores de fluxo de nêutrons foram empregados fragmentos de uma liga de Ni-Cr com massa aproximada de $10 \mathrm{mg}$ (França et al. 2003).

As amostras, materiais de referência certificados e monitores de fluxo foram irradiados com nêutrons térmicos por período de 8 horas no reator nuclear de pesquisa IEAR1m do Instituto de Pesquisas Energéticas e Nucleares, da Comissão Nacional de Energia Nuclear (IPEN/CNEN), São Paulo. A medição da radioatividade induzida foi realizada no Laboratório de Radioisótopos (LRi - CENA/USP) por meio de detectores de germânio hiperpuro, sendo dois coaxiais, com 45 e 50\% de eficiência relativa, e um do tipo poço. Após a análise dos espectros de radiação gama, foram calculadas as concentrações dos elementos químicos nas amostras e nos materiais de referência certificados por método paramétrico $\mathrm{k}_{0}$ (Bacchi et al. 2000) a partir do pacote computacional Quantu (Bacchi et al. 2003). 
A avaliação das epífitas ocorreu pela diversidade de elementos químicos encontrados e a acumulação nas folhas. O método utilizado para medir a acumulação refere-se à computação de índices variando de 0 a 1 conforme comparação entre a concentração do elemento químico $i$ para a espécie $j$ e a concentração média do elemento químico $i\left(C_{i j} \geq \bar{x}_{i}\right.$, índice $=1 ; C_{i j}<\bar{x}_{i}$, índice $\left.=0\right)$.

Empregando a função "Resampling Stats" do software EXCEL, foi realizada a aleatorização com substituição (Bootstrap) dos índices de acumulação obtidos (Manly 2004). De maneira resumida, a partir da geração de 10000 amostras simuladas, o método permite a realização de inferência sobre a média populacional e o cálculo de intervalos de confiança (95\%).

\section{Resultados e Discussão}

Os resultados de concentração dos elementos químicos determinados nos materiais de referência certificados estão dentro das faixas de incerteza (Tabela 2), comprovando a qualidade do procedimento analítico adotado para a determinação de 16 elementos químicos nas amostras.

As concentrações encontradas nas folhas estão na Tabela 3. Concentração de K na ordem de $40000 \mathrm{mg} \mathrm{kg}^{-1}$ foi determinada em folhas da espécie Billbergia amoema, enquanto a máxima concentração observada em folhas de espécies arbóreas coletadas na área foi 25000 mg kg-1 (França et al. 2005). Este fato é indicativo de considerável fonte atmosférica do elemento para o ecossistema. Contudo, a concentração de K está, em geral, na faixa esperada de 5000 a $34000 \mathrm{mg} \mathrm{kg}^{-1}$ proposta por Markert (1998).

As espécies Aechmea coelestis, Nidularium cf. inocentii, Nidularium krisgreeniae e Vriesea carinata apresentaram altas concentrações de $\mathrm{Br}$, enquanto concentração elevada de Na foi detectada nas espécies Canistropsis billbergioides e Vriesea vagans. As altas concentrações de $\mathrm{Br}$ e $\mathrm{Na}$ podem ser explicadas pela influência marinha (França et al. 2004), pois a parcela permanente está na vertente atlântica da Serra do Paranapiacaba, e há a predominância de ventos oceânicos. Verifica-se concentração acima de $40 \mathrm{mg} \mathrm{kg}^{-1}$ do elemento $\mathrm{Zn}$ na espécie $C$. billbergioides, podendo destacar poluição atmosférica já que o elemento, na maioria das vezes, possui origem antrópica (Wedepohl 1970). Elementos químicos de importância ambiental como Ba, Hg e Se também foram encontrados nas folhas de $C$. billbergioides, assim como nas folhas das espécies arbóreas da parcela (Ferrari et al. 2003).

A espécie Canistropsis billbergioides apresentou o maior índice de acumulação de elementos químicos em suas folhas (Figura 1). Após refinamento, pela simulação de 10000 amostras “bootstrap” (Figura 2), a espécie Billbergia amoema também apresentou elevado índice de acumulação.
Este tipo de procedimento estatístico pode ser aplicado somente para espécies com mais de dois indivíduos analisados. Embora B. amoema seja uma espécie de bromélia tipo tanque (reservatório de água), não foi encontrada relação entre essa característica e a acumulação de elementos químicos nas demais espécies tanque (por exemplo, Nidularium sp. e Aechmea sp.). Escolheu-se C. billbergioides devido à sua abundância e facilidade na coleta da espécie no sub bosque da floresta.

Por ser freqüentemente utilizada em estudos de biomonitoração, a espécie Tillandsia usneoides foi comparada à espécie selecionada $C$. billbergioides (Tabela 4). Os resultados de T. usneoides referentes ao indivíduo controle cultivado em casa de vegetação do Instituto de Botânica/SMA de São Paulo, ou seja, material desprovido de qualquer tipo de contaminação (Figueiredo et al. 2004), mostraram concentrações similares a C. billbergioides. Os resultados de Sc indicam que $T$. usneoides não foi lavada, uma vez que este elemento químico é considerado traçador de terra contaminante em plantas (Wyttenbach \& Tobler 1998). A presença de terra pode contribuir para o aumento na concentração de alguns elementos. Baseando-se no fato da acumulação de elementos químicos ser fator fundamental para a elegibilidade de um organismo biomonitor, C. billbergioides pode potencialmente ser utilizada como organismo biomonitor na parcela permanente do PECB, produzindo resultados comparáveis a T. usneoides.

Concluindo, dentre as espécies de epífitas avaliadas, Canistropsis billbergioides mostrou-se promissora para a biomonitoração de elementos químicos na Mata Atlântica, tendo como vantagens o fato de ser nativa da região e a sua abundância no sub bosque.

\section{Agradecimento}

Os autores agradecem à Fundação de Amparo à Pesquisa do Estado de São Paulo-FAPESP pelo auxílio concedido (Processo 04/11023-8).

\section{Referências Bibliográficas}

BACCHI, M.A., FERNANDES, E.A.N., OLIVEIRA, H. 2000. Brazilian experience on $\mathrm{k}_{0}$ standardized neutron activation analysis. J. Radioanal. Nucl. Chem. 245:217-222.

BACCHI, M.A., FERNANDES, E.A.N., OLIVEIRA, H. 2003. Quantu - design and development of a software package dedicated to $\mathrm{k}_{0}$-standardized NAA. J. Radioanal. Nucl. Chem. 257:577-582.

BREIER, T.B., SEMIR, J., RODRIGUES, R.R. 2002. Diversidade e estrutura do componente epifítico vascular em quatro formações florestais do Estado de São Paulo. In III Simpósio do Programa Biota-Fapesp. Apresentações orais. Universidade Federal de São Carlos, São Carlos, p.289-291. 
CALASANS, C.F., MALM, O. 1997. Elemental mercury contamination survey in a chlor-alkali plant by the use of transplanted Spanish moss, Tillandsia usneoides (L.). Sci. Total Environ. 208:165-177.

FERRARI, A.A., DE NADAI FERNANDES, E.A., FRANÇA, E.J., BACCHI, M.A. 2003. Influência da contaminação superficial nos teores de mercúrio em folhas de espécies arbóreas da Mata Atlântica. In $11^{\circ}$ Simpósio Internacional de Iniciação Científica da Universidade de São Paulo. Resumos. Universidade de São Paulo, Piracicaba, CD-Rom.

FIGUEIREDO, A.M.G., SAIKI, M., TICIANELLI, R.B., DOMINGOS, M., ALVES, E.S., MARKET, B. 2001. Determination of trace elements in Tillandsia usneoides by neutron activation analysis for environmental biomonitoring. J. Radioanal. Nucl. Chem. 249:391-395.

FIGUEIREDO, A.M.G., ALCALÁ, A.L., TICIANELLI, R.B., DOMINGOS, M., SAIKI, M. 2004. The use of Tillandsia usneoides L. as bioindicator of air pollution in São Paulo, Brazil. J. Radioanal. Nucl. Chem. 259:59-63.

FRANÇA, E.J., DE NADAI FERNANDES, E.A., BACCHI, M.A. 2003. Ni-Cr alloy as neutron flux monitor: composition and homogeneity assessment by NAA. J. Radioanal. Nucl. Chem. 257:113-115.

FRANÇA, E.J., DE NADAI FERNANDES, E.A., BACCHI, M.A., SAIKI, M. 2004. Native trees as biomonitors of chemical elements in the biodiversity conservations of the Atlantic Forest. J. Atmos. Chem. 49:579-592.

FRANÇA, E.J., DE NADAI FERNANDES, E.A., BACCHI, M.A., RODRIGUES. R.R., VERBURG T.G 2005. Inorganic chemical elements of native trees of the Atlantic Forest. Environ. Monit. Assess. 102:349-357.

LUOMA, S.N., RAINBOW, P.S. 2005. Why is metal bioaccumulation so variable? Biodynamics as a unifying concept. Environ. Sci. Tech. 39(7): 1921-1931.

MANLY, B. F. J. 2004. Randomization, bootstrap and Monte Carlo methods in Biology, New Zealand: University of Otago, 330p.

MARKERT, B. 1991. Inorganic chemical investigations in the Forest Biosphere Reserve near Kalinin, USSR. I. Mosses and peat profiles as bioindicators for different chemical elements. Vegetatio. 95:127-135.

MARKERT, B. 1998. Distribution and biogeochemistry of inorganic chemicals in the environment. In: SHÜÜRMANN, G., MARKERT, B. (eds) Ecotoxicology. John Wiley \& Sons, Amsterdam, p.165-222.

PYATT, F.B., GRATTAN, J.P., LACY, D., PYATT, A.J., SEAWARD, M.R.D. 1999. Comparative effective of Tillandsia usneoides L. and Parmotrema praesorediosum (NYL.) Hale as bioindicators of atmospheric pollution in Lousiana (USA). Water, Air, Soil Pollut. 111:317-326.
WEDEPOHL, K.H. 1970. Handbook of Geochemistry - II. Springer Verlag, Berlim-Heidenberg.

WOLTERBEEK, B. 2002. Biomonitoring of trace element air pollution: principles, possibilities and perspectives. Environ. Pollut. (Amsterdam, Neth.). 120:11-21.

WYTTENBACH,A., TOBLER, L. 1998. Effect of surface contamination on results of plant analysis. Commun. Soil Sci. Plant Anal. 29:809-823.

Título: Seleção de epífitas acumuladoras de elementos químicos na Mata Atlântica

Autores: Camila Elias, Elisabete A. De Nadai Fernandes, Elvis Joacir De França \& Márcio A. Bacchi

Biota Neotropica, Vol. 6 ( number 1): 2006

http://www.biotaneotropica.org.br/v6n1/pt/ abstract?article+bn02106012006

Recebido em 11/08/05 - Recebido em 15/01/2006 Publicado em 17/02/06

ISSN 1676-0603 


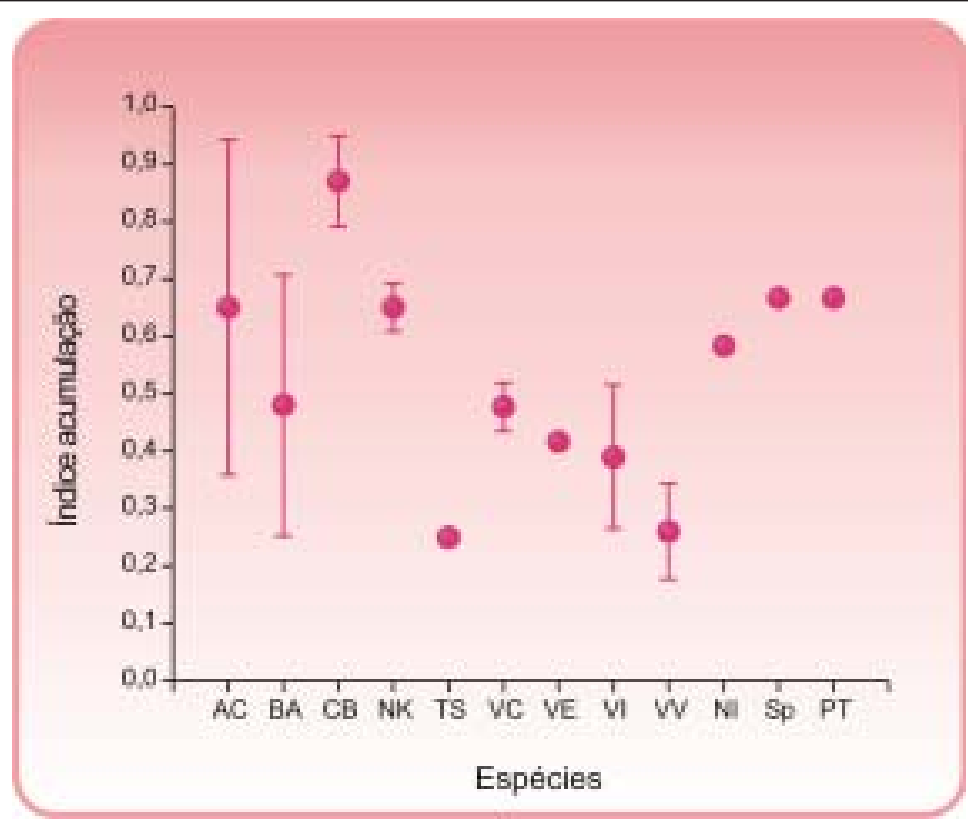

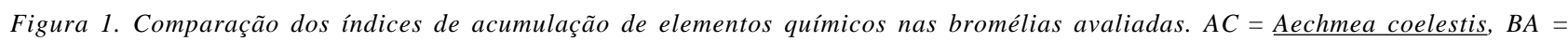
Billbergia amoema,$C B=$ Canistropsis billbergioides, $S p=$ espécie não determinada, $N I=$ Nidularium $c f$. inocentii, $N K=\underline{\text { Nidularium }}$

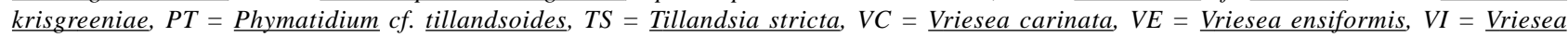
incurvata, $V V=\underline{\text { Vriesia vagans }}$

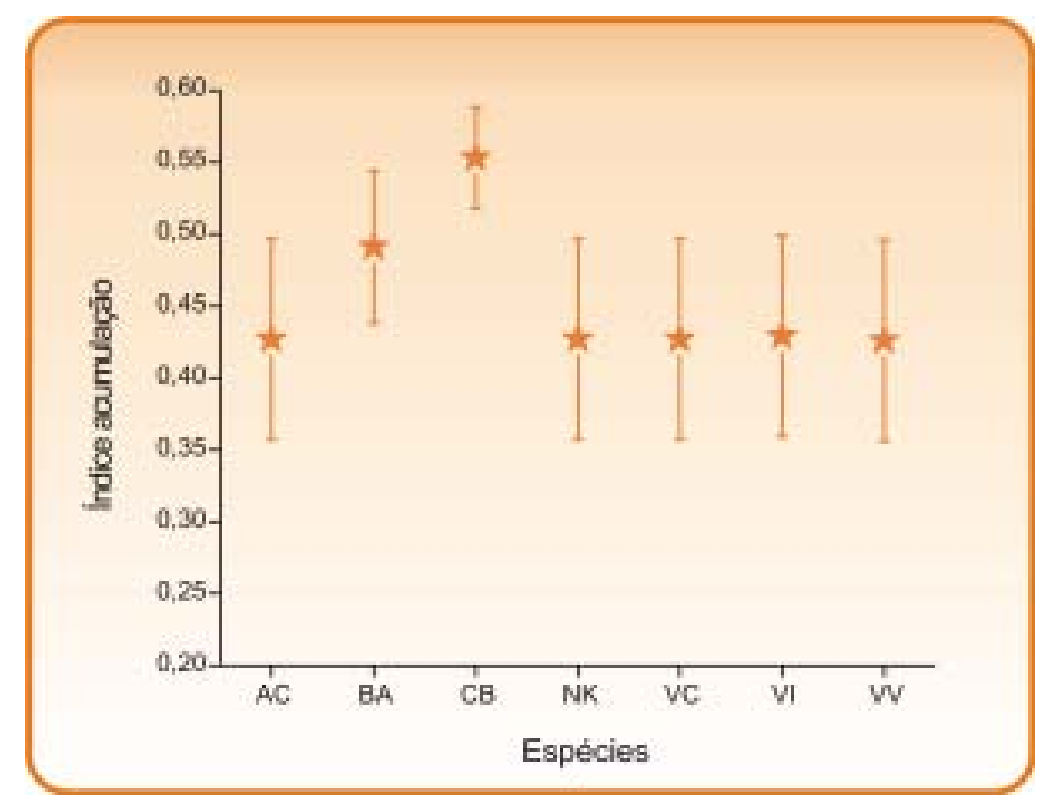

Figura 2. Índices de acumulação e seus respectivos intervalos de confiança (95\%) estimados pelo método Bootstrap (iterações $=10000)$. $A C=\underline{\text { Aechmea coelestis }}, B A=\underline{\text { Billbergia amoema }}, C B=\underline{\text { Canistropsis billbergioides }}, N K=\underline{\text { Nidularium krisgreeniae }}$, VC $=\underline{\text { Vriesea carinata }}$, $V I=\underline{\text { Vriesea incurvata }}, V V=\underline{\text { Vriesia vagans. }}$. 
Elias, C.; De Nadai Fernandes, E.A.; França, E.J. and Bacchi, M.A. - Biota Neotropica, v6 (n1) - BN02106012006

Tabela 1. Espécies coletadas na parcela permanente do Parque Estadual Carlos Botelho

\begin{tabular}{|c|c|c|c|}
\hline Espécie & Identificação & $\begin{array}{l}\text { Número de } \\
\text { replicatas }\end{array}$ & Ilustração \\
\hline Aechmea coelestis & $\mathrm{AC}$ & 2 & \\
\hline Billbergia amoema & BA & 3 & \\
\hline Canistropsis billbergioides & $\mathrm{CB}$ & 5 & \\
\hline Espécie não determinada & $\mathrm{Sp}$ & 1 & o disponive \\
\hline Nidularium cf. inocentii & $\mathrm{NI}$ & 1 & \\
\hline Nidularium krisgreeniae & NK & 2 & \\
\hline Phymatidium cf. tillandsoides & PT & 1 & \\
\hline Tillandsia stricta & TS & 1 & \\
\hline Vriesea carinata & $\mathrm{VC}$ & 2 & \\
\hline Vriesea ensiformis & $\mathrm{VE}$ & 1 & \\
\hline Vriesea incurvata & VI & 2 & \\
\hline Vriesea vagans & VV & 2 & \\
\hline
\end{tabular}

http://www.biotaneotropica.org.br 
Tabela 2. Concentrações $\left(\mathrm{mg} \mathrm{kg}^{-1}\right)$ obtidas e certificadas dos elementos químicos para os materiais de referência certificados.

\begin{tabular}{ccccc}
\hline & \multicolumn{2}{c}{ IAEA-336 } & \multicolumn{2}{c}{ INCT-TL-1 } \\
& \multicolumn{2}{c}{ Lichen } & Obtida Leaves & Certificada \\
\cline { 2 - 5 } & Obtida & Certificada & $42 \pm 4$ & $43,2 \pm 3,9$ \\
\hline $\mathrm{Ba}$ & $9 \pm 5$ & $5,3-7,5$ & $13,3 \pm 0,6$ & $12,3 \pm 1,0$ \\
$\mathrm{Br}$ & $13,7 \pm 0,6$ & $11,2-14,6$ & $6320 \pm 370$ & $5820 \pm 520$ \\
$\mathrm{Ca}$ & $2790 \pm 190$ & - & $0,85 \pm 0,07$ & $0,79 \pm 0,08$ \\
$\mathrm{Ce}$ & $1,48 \pm 0,10$ & $1,11-1,45$ & $0,39 \pm 0,02$ & $0,387 \pm 0,04$ \\
$\mathrm{Co}$ & $0,39 \pm 0,03$ & $0,24-0,34$ & $3,7 \pm 0,2$ & $3,61 \pm 0,37$ \\
$\mathrm{Cs}$ & $0,114 \pm 0,014$ & $0,097-0,123$ & $534 \pm 16$ & $432 *$ \\
$\mathrm{Fe}$ & $445 \pm 12$ & $380-480$ & $<0,078$ & $0,005 \quad 0,001$ \\
$\mathrm{Hg}$ & $0,13 \pm 0,03$ & $0,16-0,24$ & $17600 \pm 700$ & $17000 \pm 1200$ \\
$\mathrm{~K}$ & $1970 \pm 180$ & $1640-2040$ & $22 \pm 2$ & $24,7 \pm 3,2$ \\
$\mathrm{Na}$ & $342 \pm 10$ & $280-360$ & $88 \pm 4$ & $81,5 \pm 6,5$ \\
$\mathrm{Rb}$ & $2,0 \pm 0,2$ & $1,54-1,98$ & $0,26 \pm 0,01$ & $0,266 \pm 0,02$ \\
$\mathrm{Sc}$ & $0,187 \pm 0,007$ & $0,15-0,19$ & - & $0,076 *$ \\
$\mathrm{Se}$ & $0,28 \pm 0,04$ & $0,18-0,26$ & $0,154 \pm 0,012$ & $0,177 \pm 0,02$ \\
$\mathrm{Sm}$ & $0,123 \pm 0,013$ & $0,092-0,12$ & $32 \pm 6$ & $20,8 \pm 1,7$ \\
$\mathrm{Sr}$ & $16 \pm 4$ & $8,2-10,4$ & $35,7 \pm 1,1$ & $34,7 \pm 2,7$ \\
$\mathrm{Zn}$ & $31,5 \pm 1,0$ & $27-33,8$ & & \\
\hline
\end{tabular}

* Valor de concentração não certificado 
Tabela 3. Concentrações e respectivas incertezas expandidas $(k=2)$ dos elementos químicos encontrados nas folhas. AC $=$ Aechmea coelestis, $B A=$ Billbergia amoema, $C B=$ Canistropsis billbergioides, $S p=$ espécie não determinada, $N I=$ Nidularium $c f$. inocentii, $N K$ $=$ Nidularium krisgreeniae, $P T=$ Phymatidium $c f$. tillandsoides, $T S=$ Tillandsia stricta, $V C=$ Vriesea carinata, $V E=$ Vriesea ensiformis, $V I=$ Vriesea incurvata, $V V=$ Vriesia vagans

\begin{tabular}{|c|c|c|c|c|c|c|c|c|c|c|}
\hline \multirow{2}{*}{ Espécie } & $\mathrm{Ba}$ & $\mathrm{Br}$ & $\mathrm{Ca}$ & \multicolumn{2}{|c|}{$\mathrm{Ce}$} & \multicolumn{2}{|r|}{ Co } & \multirow{2}{*}{$\frac{\mathrm{Cs}}{\mathrm{mg} \mathrm{kg^{-1 }}}$} & \multirow{2}{*}{$\begin{array}{c}\mathrm{Fe} \\
m g \mathrm{~kg}^{-1}\end{array}$} & \multirow{2}{*}{$\begin{array}{c}\mathrm{Hg} \\
m g \mathrm{~kg}^{-1}\end{array}$} \\
\hline & $m g \mathrm{~kg}^{-1}$ & $m g \mathrm{~kg}^{-1}$ & $g \mathrm{~kg}^{-1}$ & \multicolumn{2}{|c|}{$m g \mathrm{~kg}^{-1}$} & \multicolumn{2}{|c|}{$m g \mathrm{~kg}^{-1}$} & & & \\
\hline AC & $24 \pm 5$ & $30,8 \pm 1,4$ & $7,5 \pm 0,5$ & \multicolumn{2}{|c|}{$0,17 \pm 0,08$} & \multicolumn{2}{|c|}{$0,69 \pm 0,08$} & $0,29 \pm 0,03$ & $62 \pm 3$ & $<0,035$ \\
\hline AC & $10 \pm 2$ & $11,0 \pm 0,5$ & $2,6 \pm 0,2$ & \multicolumn{2}{|c|}{$0,13 \pm 0,03$} & \multicolumn{2}{|c|}{$0,073 \pm 0,007$} & $0,061 \pm 0,005$ & $133 \pm 4$ & $0,022 \pm 0,012$ \\
\hline BA & $16 \pm 3$ & $21,4 \pm 0,9$ & $5,7 \pm 0,3$ & 0,19 & $=0,03$ & & $\pm 0,02$ & $0,122 \pm 0,015$ & $68 \pm 2$ & $<0,035$ \\
\hline BA & $7 \pm 3$ & $4,3 \pm 0,2$ & $1,5 \pm 0,1$ & 0,33 & $=0,04$ & $0,0 €$ & $2 \pm 0,005$ & $0,055 \pm 0,006$ & $200 \pm 6$ & $0,017 \pm 0,014$ \\
\hline BA & $28 \pm 2$ & $15,2 \pm 0,7$ & $8,1 \pm 0,4$ & 0,25 & 0,04 & 0,02 & $9 \pm 0,003$ & $0,084 \pm 0,008$ & $80 \pm 3$ & $0,027 \pm 0,017$ \\
\hline $\mathrm{CB}$ & $14 \pm 2$ & $10,6 \pm 0,5$ & $7,3 \pm 0,4$ & & 08 & 0,14 & $2 \pm 0,009$ & $0,137 \pm 0,010$ & $57 \pm 2$ & $0,03 \pm 0,02$ \\
\hline $\mathrm{CB}$ & $45 \pm 4$ & $22,0 \pm 1,1$ & $5,3 \pm 0,3$ & 0,16 & 0,02 & 0,22 & $9 \pm 0,013$ & $0,177 \pm 0,017$ & $148 \pm 4$ & $<0,035$ \\
\hline $\mathrm{CB}$ & $20 \pm 3$ & $12,7 \pm 0,6$ & $5,5 \pm 0,3$ & 0,10 & $=0,03$ & 0,16 & $4 \pm 0,011$ & $0,098 \pm 0,009$ & $133 \pm 4$ & $<0,035$ \\
\hline CB & $11 \pm 2$ & $21,2 \pm 1,0$ & $4,0 \pm 0,3$ & 0,12 & $=0,03$ & 0,08 & $6 \pm 0,006$ & $0,186 \pm 0,014$ & $123 \pm 5$ & $0,031 \pm 0,016$ \\
\hline $\mathrm{CB}$ & $21 \pm 2$ & $18,2 \pm 0,8$ & $7,5 \pm 0,4$ & 1,01 & 0,07 & 0,18 & $1 \pm 0,011$ & $0,111 \pm 0,010$ & $378 \pm 10$ & $0,063 \pm 0,018$ \\
\hline NI & $13 \pm 2$ & $12,2 \pm 0,6$ & $5,2 \pm 0,3$ & & 08 & 0,01 & $7 \pm 0,003$ & $0,146 \pm 0,011$ & $76 \pm 3$ & $0,035 \pm 0,015$ \\
\hline NK & $11 \pm 4$ & $24,4 \pm 1,1$ & $3,9 \pm 0,2$ & 0,17 & 0,04 & $0,0 \varepsilon$ & $5 \pm 0,006$ & $0,23 \pm 0,02$ & $70 \pm 3$ & $0,04 \pm 0,02$ \\
\hline NK & $14 \pm 2$ & $17,1 \pm 0,8$ & $3,3 \pm 0,3$ & 0,07 & $=0,06$ & 0,12 & $2 \pm 0,012$ & $0,083 \pm 0,007$ & $67 \pm 2$ & $0,026 \pm 0,015$ \\
\hline $\mathrm{PT}$ & $52 \pm 5$ & $5,2 \pm 0,3$ & $4,6 \pm 0,3$ & 0,48 & 0,08 & 0,10 & $1 \pm 0,010$ & $0,48 \pm 0,03$ & $196 \pm 7$ & $0,07 \pm 0,03$ \\
\hline Sp & $11 \pm 5$ & $29,6 \pm 1,3$ & $2,1 \pm 0,2$ & 0,10 & 0,06 & & $1 \pm 0,03$ & $0,174 \pm 0,013$ & $95 \pm 3$ & $0,05 \pm 0,02$ \\
\hline TS & $15 \pm 2$ & $11,8 \pm 0,6$ & $3,5 \pm 0,2$ & 0,63 & $=0,05$ & 0,08 & $1 \pm 0,006$ & $0,074 \pm 0,007$ & $123 \pm 4$ & $<0,035$ \\
\hline VC & $11 \pm 2$ & $12,6 \pm 0,6$ & $1,6 \pm 0,2$ & 0,18 & 0,08 & 0,20 & $6 \pm 0,012$ & $0,252 \pm 0,019$ & $181 \pm 7$ & $0,048 \pm 0,019$ \\
\hline VC & $5 \pm 1$ & $23,0 \pm 1,0$ & $2,0 \pm 0,1$ & 0,15 & $=0,03$ & 0,14 & $2 \pm 0,009$ & $0,113 \pm 0,011$ & $173 \pm 6$ & $0,049 \pm 0,017$ \\
\hline $\mathrm{VE}$ & $14 \pm 6$ & $13,8 \pm 0,6$ & $2,3 \pm 0,2$ & 0,12 & 0,04 & 0,15 & $7 \pm 0,010$ & $0,063 \pm 0,006$ & $166 \pm 5$ & $0,06 \pm 0,02$ \\
\hline VI & $10 \pm 2$ & $7,3 \pm 0,4$ & $3,7 \pm 0,2$ & 0,08 & 0,02 & 0,23 & $3 \pm 0,013$ & $0,145 \pm 0,011$ & $178 \pm 5$ & $<0,035$ \\
\hline VI & $6 \pm 2$ & $13,0 \pm 0,6$ & $1,7 \pm 0,1$ & 0,07 & 0,03 & 0,12 & $1 \pm 0,007$ & $0,244 \pm 0,018$ & $178 \pm 5$ & $0,034 \pm 0,02$ \\
\hline VV & $36 \pm 3$ & $7,5 \pm 0,4$ & $1,9 \pm 0,2$ & 0,16 & 0,04 & 0,06 & $5 \pm 0,006$ & $0,056 \pm 0,011$ & $112 \pm 4$ & $0,054 \pm 0,02$ \\
\hline VV & $16 \pm 2$ & $9,6 \pm 0,5$ & $2,5 \pm 0,2$ & 0,15 & 0,02 & 0,05 & $7 \pm 0,004$ & $0,122 \pm 0,012$ & $231 \pm 7$ & $0,048 \pm 0,01$ \\
\hline Espécie & $\mathrm{K}$ & $\mathrm{Na}$ & $\mathrm{R}$ & & Sc & & Se & $\mathrm{Sm}$ & $\mathrm{Sr}$ & $\mathrm{Zn}$ \\
\hline Especie & $g \mathrm{~kg}^{-1}$ & $g \mathrm{~kg}^{-1}$ & $m g$ & & $\mu g k$ & & $m g \mathrm{~kg}^{-1}$ & $\mu g \mathrm{~kg}^{-1}$ & $m g \mathrm{~kg}^{-1}$ & $m g \mathrm{~kg}^{-1}$ \\
\hline $\mathrm{AC}$ & $35,8 \pm 1,4$ & $5,03 \pm 0,14$ & 68 & & $5,2=$ & & $<0,107$ & $4,5 \pm 1,7$ & $156 \pm 12$ & $24,5 \pm 1,0$ \\
\hline $\mathrm{AC}$ & $23,3 \pm 0,8$ & $4,72 \pm 0,17$ & 45 & & 8,5 & & $0,18 \pm 0,06$ & $8,4 \pm 1,3$ & $50 \pm 4$ & $16,8 \pm 0,6$ \\
\hline BA & $39,7 \pm 1,3$ & $2,75 \pm 0,09$ & 74 & & 4,4 & & $<0,107$ & $6,7 \pm 1,5$ & $156 \pm 9$ & $15,2 \pm 0,5$ \\
\hline BA & $3,0 \pm 0,3$ & $2,21 \pm 0,06$ & 6,5 & 0,3 & 17,6 & 0,9 & $0,08 \pm 0,03$ & $6,7 \pm 1,1$ & $25 \pm 3$ & $10,4 \pm 0,4$ \\
\hline BA & $29,2 \pm 0,8$ & $4,90 \pm 0,16$ & 45 & & 5,7 & & $<0,107$ & $7,1 \pm 1,1$ & $218 \pm 13$ & $15,6 \pm 0,6$ \\
\hline $\mathrm{CB}$ & $20,8 \pm 0,7$ & $4,03 \pm 0,13$ & 36,2 & 1,7 & 5,8 & 0,4 & $<0,107$ & $<2,9$ & $133 \pm 8$ & $13,8 \pm 0,5$ \\
\hline $\mathrm{CB}$ & $21,5 \pm 0,8$ & $8,5 \pm 0,3$ & 24,7 & 1,2 & 10,1 & 0,6 & $0,17 \pm 0,03$ & $5,5 \pm 0,8$ & $98 \pm 8$ & $27,2 \pm 1,0$ \\
\hline CB & $23,6 \pm 0,7$ & $7,1 \pm 0,3$ & 30,3 & 1,4 & $8,2=$ & 0,5 & $0,11 \pm 0,03$ & $3,5 \pm 1,7$ & $108 \pm 6$ & $33,5 \pm 1,2$ \\
\hline $\mathrm{CB}$ & $29,6 \pm 1,0$ & $5,3 \pm 0,2$ & 51 & & 5,9 & 0,4 & $0,11 \pm 0,03$ & $3,5 \pm 1,9$ & $86 \pm 6$ & $40,3 \pm 1,4$ \\
\hline $\mathrm{CB}$ & $30,2 \pm 0,9$ & $5,09 \pm 0,16$ & 48 & & 36,5 & 1,5 & $0,37 \pm 0,09$ & $20 \pm 2$ & $147 \pm 9$ & $33,9 \pm 1,0$ \\
\hline NI & $27,3 \pm 0,9$ & $2,92 \pm 0,11$ & 63 & & $2,6=$ & 0,3 & $0,21 \pm 0,08$ & $5 \pm 2$ & $166 \pm 9$ & $22,4 \pm 0,7$ \\
\hline NK & $29,4 \pm 0,9$ & $5,22 \pm 0,18$ & 79 & & $3,9=$ & 0,3 & $<0,107$ & $<2,9$ & $73 \pm 8$ & $21,9 \pm 0,8$ \\
\hline NK & $29,4 \pm 1,1$ & $4,69 \pm 0,14$ & 51 & & $3,5=$ & 0,3 & $<0,107$ & $<2,9$ & $90 \pm 7$ & $18,6 \pm 0,7$ \\
\hline $\mathrm{PT}$ & $21,1 \pm 0,8$ & $0,77 \pm 0,03$ & 76 & & 53 & & $0,37 \pm 0,09$ & $21 \pm 2$ & $81 \pm 8$ & $23,3 \pm 0,7$ \\
\hline Sp & $25,8 \pm 0,7$ & $4,35 \pm 0,15$ & 58 & & 2,9 & 0,3 & $0,14 \pm 0,03$ & $<2,9$ & $33 \pm 3$ & $19,7 \pm 0,7$ \\
\hline TS & $10,9 \pm 0,6$ & $4,82 \pm 0,16$ & 18,1 & 0,8 & 23,4 & 1,1 & $0,25 \pm 0,08$ & $17,2 \pm 1,5$ & $58 \pm 4$ & $17,7 \pm 0,6$ \\
\hline VC & $14,9 \pm 0,5$ & $4,00 \pm 0,14$ & 28,0 & 1,3 & 8,2 & 0,7 & $0,14 \pm 0,03$ & $4,0 \pm 0,9$ & $43 \pm 13$ & $37,3 \pm 1,2$ \\
\hline VC & $14,2 \pm 0,5$ & $4,75 \pm 0,16$ & 32,4 & 1,4 & $5,9=$ & & $0,12 \pm 0,02$ & $5,1 \pm 0,9$ & $43 \pm 4$ & $21,4 \pm 0,8$ \\
\hline $\mathrm{VE}$ & $14,8 \pm 0,5$ & $3,33 \pm 0,10$ & 26,9 & 1,2 & $5,0=$ & 0,4 & $0,08 \pm 0,02$ & $19 \pm 2$ & $62 \pm 6$ & $22,4 \pm 0,9$ \\
\hline VI & $6,6 \pm 0,3$ & $3,62 \pm 0,11$ & 16,2 & 0,8 & $5,6=$ & 0,4 & $0,21 \pm 0,06$ & $<2,9$ & $81 \pm 6$ & $19,5 \pm 0,6$ \\
\hline VI & $16,6 \pm 0,6$ & $2,88 \pm 0,09$ & 34,5 & 1,6 & $3,6=$ & 0,4 & $0,10 \pm 0,02$ & $2,8 \pm 0,8$ & $40 \pm 9$ & $37,0 \pm 1,3$ \\
\hline VV & $7,2 \pm 0,4$ & $2,59 \pm 0,07$ & 15,4 & 0,7 & 10,9 & 0,7 & $0,07 \pm 0,04$ & $6,0 \pm 0,7$ & $<2,8$ & $14,7 \pm 0,6$ \\
\hline VV & $8,3 \pm 0,3$ & $5,8 \pm 0,2$ & 13,2 & 0,6 & $7,8=$ & 0,5 & $0,27 \pm 0,07$ & $5,5 \pm 0,8$ & $69 \pm 4$ & $14,5 \pm 0,6$ \\
\hline
\end{tabular}


Tabela 4. Comparação entre as concentrações $\left(\mathrm{mg} \mathrm{kg}^{-1}\right)$ nas espécies de bromélias Canistropsis billbergioides e Tillandsia usneoides

\begin{tabular}{|c|c|c|c|c|c|}
\hline & \multirow{2}{*}{\multicolumn{2}{|c|}{$\begin{array}{l}\text { Canistropsis } \\
\text { billbergioides }\end{array}$}} & \multicolumn{3}{|c|}{ Tillandsia usneoides } \\
\hline & & & \multirow{2}{*}{$\begin{array}{c}\begin{array}{c}\text { Figueiredo } \\
(2004)\end{array} \\
\text { Controle }\end{array}$} & \multirow{2}{*}{$\begin{array}{c}\begin{array}{c}\text { Figueiredo } \\
\text { (2001) }\end{array} \\
\text { São Paulo }\end{array}$} & \multirow{2}{*}{$\begin{array}{c}\begin{array}{c}\text { Calasans } \\
(1997)\end{array} \\
\text { Rio de Janeiro }\end{array}$} \\
\hline & Média & Inc. & & & \\
\hline $\mathrm{Ba}$ & \multicolumn{2}{|c|}{$22 \pm 2$} & 16 & 49 & - \\
\hline $\mathrm{Br}$ & \multicolumn{2}{|c|}{$16,9 \pm 0,8$} & 10,1 & 6,43 & - \\
\hline $\mathrm{Ca}$ & \multicolumn{2}{|c|}{$5930 \pm 350$} & 3303 & - & - \\
\hline $\mathrm{Ce}$ & \multicolumn{2}{|c|}{$0,35 \pm 0,04$} & 1,4 & 5,1 & - \\
\hline Co & \multicolumn{2}{|c|}{$0,16 \pm 0,01$} & 0,61 & 1,1 & - \\
\hline $\mathrm{Fe}$ & \multicolumn{2}{|c|}{$168 \pm 5$} & 650 & 2536 & - \\
\hline $\mathrm{Hg}$ & \multicolumn{2}{|c|}{$0,043 \pm 0,019$} & - & - & 2,1 \\
\hline $\mathrm{K}$ & \multicolumn{2}{|c|}{$25100 \pm 830$} & 5850 & 4713 & - \\
\hline $\mathrm{Na}$ & \multicolumn{2}{|c|}{$6020 \pm 220$} & 421 & 601 & - \\
\hline $\mathrm{Rb}$ & \multicolumn{2}{|c|}{$38,1 \pm 1,8$} & 37 & 89 & - \\
\hline Sc & \multicolumn{2}{|c|}{$0,0133 \pm 0,0007$} & 0,13 & 0,18 & - \\
\hline Se & \multicolumn{2}{|c|}{$0,19 \pm 0,05$} & 0,25 & - & - \\
\hline Sm & \multicolumn{2}{|c|}{$0,007 \pm 0,002$} & 0,083 & 0,26 & - \\
\hline $\mathrm{Zn}$ & \multicolumn{2}{|c|}{$29,7 \pm 1,0$} & 41 & 73 & - \\
\hline
\end{tabular}

